Recepción: 17/11/2015

Evaluación: 20/11/2015

Aprobación: 28/11/2015

Artículo de Investigación Científica

\title{
OBJETOS VIRTUALES DE APRENDIZAJE Y UN LABORATORIO VIRTUAL DE QUÍMICA EN LA ENSEÑANZA DE LA LEY DE CONSERVACIÓN DE LA MASA
}

\author{
Henry Eduardo Escobar Pérez* \\ I.E.M. Eduardo Romo Rosero Pasto Nariño \\ Universidad de Nariño \\ henryesc@gmail.com \\ Luis Aníbal Benavides Burgos** \\ I.E. Agrícola de la Sabana Túquerres Nariño \\ Grupo de Investigación para el Desarrollo de la Educación \\ y la Pedagogía (GIDEP) Universidad de Nariño \\ luisanibalben@yahoo.es
}

\begin{abstract}
RESUMEN
En esta investigación exploratoria y descriptiva se evaluó la utilización de un programa guía de actividades que incluyen OVAs y el laboratorio virtual de química VLabQ, para la enseñanza de la ley de conservación de la masa a estudiantes de grado décimo, de la I.E.M. Eduardo Romo Rosero del municipio de Pasto. Se aplicó un test de Likert para determinar cambios de actitudes hacia el estudio de la química, se elaboró un test para conocer ideas previas y otro test de trece preguntas para evaluar conocimientos sobre la ley de conservación de la masa. El uso pedagógico de OVAs mejoró el interés y la motivación por las clases, el laboratorio virtual de química VLabQ al programa guía de actividades acercó a los estudiantes a la experimentación en química. En el postest de Likert las actitudes
\end{abstract}

* Químico, Docente I.E.M. Eduardo Romo Rosero, Pasto - Nariño, Docente hora cátedra Universidad de Nariño, Egresado VII promoción maestría en educación Universidad de Nariño, e-mail:henryesc@ gmail.com

** Licenciado y especialista en docencia de la Química, Magíster en docencia, Docente hora cátedra Universidad de Nariño, Grupo de Investigación para el Desarrollo de la Educación y la Pedagogía (GIDEP) Universidad de Nariño, Docente I.E. Agrícola de la Sabana Túquerres Nariño, e-mail:luisanibalben@ yahoo.es 
evaluadas se clasificaron entre moderadamente positivas y muy positivas. El pretest para conocer las ideas previas sobre la ley de conservación de la masa indican que los estudiantes escogen una respuesta con base en sus ideas previas y el estado inicial y final del sistema; en el postest se elige la respuesta con estos mismos criterios complementados con algunas ideas de lo aprendido, demostrando que las ideas previas persisten luego de la enseñanza o que no se alcanzó un nivel significativo de aprendizaje. En el postest de evaluación de conocimientos sobre la ley de conservación de la masa aumentó el porcentaje de respuestas correctas, que paso en promedio del $32 \%$ al $72,85 \%$.

Palabras clave: Programa guía, Conservación de la masa, Objeto virtual, Laboratorio virtual de química.

\title{
VIRTUAL LEARNING OBJECTS AND A VIRTUAL CHEMISTRY LABORATORY IN THE TEACHING OF THE LAW OF THE CONSERVATION OF MASS
}

\begin{abstract}
This exploratory and descriptive study evaluates the use of a programmed guide of activities that include OVA's and the virtual chemical lab VLabQ, for teaching the Law of Conservation of Mass to 10th graders in the Eduardo Rosero Institute (I.E.M) in the municipality of Pasto. A Likert Test was applied to determine changes in attitudes toward the study of Chemistry, another test was developed to determine previous ideas, and another test of thirteen questions to assess knowledge of the Law of Conservation of Mass. The pedagogical use of OVA's improved interest and motivation in classes, while the Virtual Chemical Laboratory of the VLabQ program actively guided students in an experimental approach to Chemistry. The Likert post-test, assessed attitudes were classified between moderately positive and very positive. The pre-test to observe previous ideas about the Law of Conservation of Mass indicates that students choose a response based on their previous ideas and the initial and final state of the system; the post-test is designed for students to choose answers with these same criteria, supplemented by ideas of what they had learned, showing how previous ideas persist after education, or how a significant level of learning had not been reached. The post-test evaluation of knowledge of the Law of Conservation of Mass increased the percentage of correct answers from an average of $32 \%$ to $72,85 \%$.
\end{abstract}

Key words: program guide, conservation of mass, virtual object, virtual chemistry laboratory. 


\title{
OBJETOS DE APRENDIZAGEMVIRTUAL EM UM LABORATÓRIO VIRTUAL DE QUÍMICA NA EDUCAÇÃO DA LEI DA CONSERVAÇÃO DA MASSA
}

\begin{abstract}
RESUMO
Nesta pesquisa exploratória e descritiva se avaliou um programa guia de atividades, incluindo OVAs e o laboratório de química virtual VLabQ para ensinar a lei da conservação da massa a estudantes do décimo grau da I.E.M. Eduardo Romo Rosero do município de Pasto. teste de Likert foi aplicado para determinar as mudanças nas atitudes para o estudo da química, um teste foi desenvolvido para conhecer as idéias anteriores e outro de treze perguntas do teste para avaliar o conhecimento da lei de conservação da massa. 0 uso pedagógico das OVAs melhorou o interesse e motivação pelas classes, o laboratório químico virtual VLabQ ao programa guía deatividades se aproximou aos alunos à experimentação em química. No postest de Likert as atitudes avaliadas foram classificadas moderadamente positiva e muito positiva. 0 pretest para conhecer as ideias anteriores sobre a lei da conservação de massa indicam que os alunos escolhem uma resposta com base em suas idéias anteriores e o estado inicial e final do sistema; no postest se escolhe a resposta com os mesmos critérios suplementadas com algumas idéias de aprendizagem. Demonstrando que as idéias anteriores persistem após o ensino o que não foram alcançados ao nível significativo de aprendizagem. No postest de avaliação de conhecimento sobre a lei da conservação de massa aumentou a porcentagem de acertos, aconteceu, em média, de $32 \%$ para $72,85 \%$.
\end{abstract}

Palavras-chave: Programa de Orientação, conservação da massa, objeto virtual, laboratório de química virtual. 


\section{INTRODUCCIÓN}

La enseñanza de las ciencias y en particular la enseñanza de la química aún se realizan de forma tradicional, asumiendo que enseñar es transmitir conocimientos y que aprender es repetir y memorizar. Además, se presta poca atención a la relación e impacto de la ciencia en nuestra vida cotidiana y se ha descuidado el papel que cumple el laboratorio en la enseñanza de las ciencias, siendo una de las actividades más importantes para la enseñanza de la química, porque introduce al estudiante en un ambiente en el cual conocerá procedimientos, materiales y equipos de laboratorio; contrastará la teoría con la realización de experimentos; planteará y resolverá problemas de investigación que lo acercarán a la metodología de la ciencia. Por esto, toda institución educativa debe contar con un laboratorio de química, como un espacio para la enseñanza y aprendizaje de esta ciencia, situación que no se presenta en la I.E.M. Eduardo Romo Rosero ubicada en el Corregimiento de Buesaquillo del Municipio de Pasto.

En la actualidad las TIC constituyen una importante herramienta para la educación, por la diversidad de aplicaciones informáticas disponibles en la Web para la enseñanza; sin embargo, en la I.E.M. Eduardo Romo Rosero no se han implementado proyectos de uso pedagógico de las TIC, que de incorporarse en la enseñanza de la química pueden constituirse en una herramienta fundamental para mejorar procesos de enseñanza y aprendizaje, permitiendo además la realización de prácticas de laboratorio virtuales, para acercar a los estudiantes a la experimentación en química.

El docente por su práctica pedagógica conoce que los estudiantes presentan dificultades en el aprendizaje de algunos conceptos importantes de química, entre ellos el de la ley de conservación de la masa. Posible consecuencia de que el docente explica o enseña conceptos que se tienen que memorizar sin comprensión alguna, situación que no garantiza un aprendizaje significativo. En investigaciones realizadas por Oñorbe y Sánchez ${ }^{1}$, Landau y Lastres$^{2}$, y Ozmen y Ayas $^{3}$, sobre cambios físicos, cambios químicos y la ley de conservación de la masa, se demuestra que los estudiantes creen comprender la ley de conservación de la masa, pero no aplican bien el concepto al resolver

1. A. Oñorbe y J. Sánchez, "La masa no se crea ni se destruye. ¿Estáis seguros?" Enseñanza de las Ciencias. Vol. 10. No. 2 (1992): 165-171, http://www.raco.cat/index.php/Ensenanza/article/ download/39817/93184

2. L. Landau y L. Lastres, “Cambios Químicos y Conservación de la Masa... ¿Esta todo claro?” Enseñanza de las Ciencias. Vol. 14. No. 2 (1996): 171-174, http://www.raco.cat/index.php/ensenanza/article/ viewFile/21446/93409

3. Haluk Ozmen y Alipasa Ayas, "'Students' difficulties in understanding of the conservation of matter in open and closed-system chemical reactions". Chemistry Education: Research And Practice. Vol. 4. No. 3 (2003): 279-290, http://www.uoi.gr/cerp/2003_0ctober/pdf/040zmen.pdf 
pregunta en las que se aplica esta ley, como también que no interpretan bien los cambios físicos y químicos de la materia. Evidenciándose según estos autores que el aprendizaje de algunos conceptos básicos de química es memorístico o no es significativo, que no se comprende la ley de conservación de la masa, que se nota la presencia de concepciones alternativas que son resistentes a la enseñanza y se consideran necesario un replanteamiento en la enseñanza de estos fundamentos de química, porque solo su verdadera compresión garantizaría el aprendizaje y aplicación posterior el estudio de otros conceptos de química. Una alternativa distinta a la enseñanza tradicional es la visión constructivista para la enseñanza de las ciencias que plantea: que aprender es construye significados, comprender es establecer relaciones, se aprende desde las ideas previas, el estudiante es un sujeto activo en su aprendizaje y el docente es un acompañante y orientador de este proceso; aspectos que se contemplan en el modelo de enseñanza por investigación dirigida y el programa guía de actividades.

La carencia de un laboratorio de química, la posibilidad de utilizar las ventajas que ofrecen las TIC para la enseñanza de la química, y el abordar dificultades en el aprendizaje de conceptos de química desde el constructivismo, condujeron a un proyecto de investigación exploratoria y descriptiva en el cual se evaluó la utilización de un programa guía de actividades que incluye OVAs y el LVQ VLabQ para la enseñanza de la ley de conservación de la masa, en estudiantes de grado décimo de la I.E.M. Eduardo Romo Rosero del Corregimiento de Buesaquillo Municipio de Pasto. Proyecto en el que buscó aprendizajes significativos en niveles de complejidad creciente, se abordó las temáticas de estudio incluyendo relaciones con la vida cotidiana, la participación activa de los estudiantes, el trabajo colaborativo en el aula y la realización de una evaluación integral, que son algunos de los deberes del docente según el Ministerio de Educación Nacional.

El trabajo de investigación está enmarcado en el constructivismo, que plantea que el individuo construye su propio conocimiento y el papel del docente no es trasmitir conocimientos, sino acompañar y orientar al alumno en su proceso de construcción de conocimiento y crear condiciones para que este proceso se pueda dar ${ }^{4}$. El constructivismo ha sido calificado como el aporte más importante en la enseñanza de las ciencias en las últimas décadas, que según Resnick (citado por Daniel Gil Pérez ${ }^{5}$ ), se resumen en los siguientes

4. Carlos Hernández, "Aproximación a un estado del arte de la enseñanza de las ciencias en Colombia". En Estados del Arte de la Investigación en Educación y Pedagogía en Colombia. Bogotá: ICFES, Colciencias, Sicolpe, 2001.1-72, http://www.socolpe.org/data/public/libros/InvestigacionPedagogia/2-1 Ciencias-Estado del Arte.pdf

5. Daniel Gil Pérez, "Contribución de la historia y de la filosofía de las ciencias al desarrollo de un modelo de enseñanza/aprendizaje como investigación". Enseñanza de las Ciencias, Vol. 11. No. 2 (1993): 197212, http://ddd.uab.cat/pub/edlc/02124521v11n2p197.pdf 
tres principios: quienes aprenden construyen significados, no reproducen simplemente lo que se les enseña; comprender supone establecer relaciones; y que todo aprendizaje depende de conocimientos previos. Exigiendo según Daniel Gil Pérez (citado por Hernández ${ }^{6}$ ) al docente constructivista las siguientes competencias: conocer la materia a enseñar; conocer y cuestionar el pensamiento docente de sentido común; adquirir conocimiento teóricos sobre el aprendizaje de las ciencias; analizar críticamente la enseñanza habitual; preparar actividades que generen un aprendizaje significativo; saber dirigir y evaluar las actividades de los alumnos.

Para $\mathrm{Pozo}^{7}$ uno de los modelos de enseñanza de las ciencias de carácter constructivista que se considera mejor aplica los postulados constructivistas, es la enseñanza mediante investigación dirigida, que busca lograr cambios actitudinales y conceptuales en los alumnos, situándolos en un contexto de actividad similar al que vive un científico, pero bajo la atenta dirección del profesor quien actuaría como un director de investigaciones. En este modelo didáctico ${ }^{8}$, el estudiante es un sujeto activo con conocimientos previos, que construye su conocimiento desde el desarrollo de procesos de investigación, y el docente es quien plantea problemas significativos para sus alumnos que pueden ser resueltos desde la ciencia escolar. Campanario y Moya ${ }^{9}$ presentan una serie de estrategias que se pueden aplicar para desarrollar el modelo de investigación dirigida, entre las que se encuentran plantear situaciones problemáticas que generen interés, análisis grupal de las situaciones problemáticas para delimitar el problema, emisión de hipótesis, elaboración de estrategias posibles de resolución y análisis, comparación de resultados, profundización de los temas y establecer relaciones entre ciencia tecnología y sociedad. Según lo planteado por Gil (citado por Campanario y Moya ${ }^{\mathbf{1 0}}$ ) a lo largo de todo este proceso se puede presentar un cambio conceptual como resultado de resolver el problema de interés, al enfrentar al alumno a las distintas estrategias planteadas en la investigación escolar, que además, debe producir esquemas, memorias, mapas conceptuales que permitan concebir nuevos problemas. Lo que se busca mediante la investigación dirigida es acercar al estudiante al trabajo científico y sus resultados, replicando para ello investigaciones realizadas por otros, abordando problemas conocidos por quienes dirigen su trabajo; se incrementa el nivel de participación y la

\footnotetext{
6. Hernández, “Aproximación a un estado del arte de la enseñanza de las ciencias en Colombia”, 2.

7. Juan Pozo y Mario Gómez, Aprender y enseñar ciencias, 5ta. edición (Madrid: Ediciones Morata, S.L., 2006).

8. Francisco Ruiz, "Modelos didácticos para la enseñanza de las ciencias naturales". Revista latinoamericana de estudios educativos, Vol. 3. No. 2 (2007): 41-60, http://www.redalyc.org/articulo. oa?id=134112600004

9. Juan Campanario y Aida Moya, “¿Cómo enseñar ciencias? principales tendencias y propuestas". Enseñanza de las ciencias, Vol. 17 No. 2 (1999): 179-192, http://www2.uah.es/jmc/an11.pdf

10. Campanario y Moya, “¿Cómo enseñar ciencias? principales tendencias y propuestas”, 3.
} 
creatividad, se favorece el trabajo en grupo, la interacción entre grupos, y el compartir información, como se hace en una comunidad científica ${ }^{11}$.

Para la enseñanza de la ley de conservación de la masa desde el modelo de enseñanza de las ciencias por investigación dirigida se diseñaron cuatro programas guía de actividades, que para Gil y Martínez ${ }^{12}$ son una aplicación del modelo constructivista de enseñanza de las ciencias, que busca favorecer la construcción de conocimiento por parte de los estudiantes, al familiarizarlos con algunas características del trabajo científico. Los programas guía describen una secuencia de enseñanza en términos genéricos, relacionando el conjunto de actividades que se incluye en ella y posibles alternativas de trabajos adicionales y están abiertos a posibles modificaciones, dependiendo de los resultados que se obtenga de su aplicación (Campanario y Moya ${ }^{13}$. Las actividades de un programa guía pretenden enfrentar al alumno a situaciones que se pueden presentar en un proceso de investigación replicando investigaciones realizadas por otros y conocidas por quien dirige su trabajo; entre las ventajas de este modelo didáctico están el trabajo colectivo en pequeños grupos, que incrementan el nivel de participación, la creatividad y el intercambio de información $\mathrm{n}^{\mathbf{1 4}}$. Los programas guía de actividades se tienen que diseñar con una metodología activa para el alumno, que le permitan elaborar conocimientos, explorar alternativas y superar la asimilación de conocimientos elaborados y que además posibilite la interacción entre compañeros; las actividades deben cubrir el contenido del tema, tener una lógica interna que eviten un aprendizaje inconexo y acercar al alumno a la metodología científica; un programa guía puede experimentar modificaciones y ajustes en el transcurso de su aplicación, porque su elaboración se convierte en un trabajo de investigación y didáctica ${ }^{15}$.

Teniendo en cuenta la carencia de laboratorio de química en la I.E.M. Eduardo Romo Rosero y la importancia que tiene la experimentación en la enseñanza de la química, el en cuarto taller del programa guía de actividades se incluye la realización de un práctica de laboratorio virtual sobre la ley de conservación de la masa, para acercar a los estudiantes al laboratorio de química y la experimentación. Porque teniendo en cuenta a autores como

11. Gil, "Contribución de la historia y de la filosofía de las ciencias al desarrollo de un modelo de enseñanza/aprendizaje como investigación", 2.

12. Daniel Gil Pérez y Joaquín Martínez, "Los programas-guía de actividades: una concreción del modelo constructivista de aprendizaje de las ciencias". Investigación en la escuela, Vol. 3 (1987): 3-12, http:// www.investigacionenlaescuela.es/articulos/3/R3_1.pdf

13. Campanario y Moya, “¿Cómo enseñar ciencias? principales tendencias y propuestas”, 3.

14. Gil, "Contribución de la historia y de la filosofía de las ciencias al desarrollo de un modelo de enseñanza/aprendizaje como investigación", 2.

15. José Hierrezuelo, Eduardo Molina y Rafael Yus, "Una nueva generación de materiales curriculares para la enseñanza de la ciencia: los programas-guía de actividades”. Revista de Educación, No. 295 (1991): 463-486, http://www.mecd.gob.es/dctm/revista-de-educacion/articulosre295/re29514. pdf?documentId=0901e72b813578ea 
Caamaño ${ }^{16}$ y Hodson ${ }^{17}$ los trabajos prácticos permiten al estudiante la observación y la interpretación de fenómenos que son objetos de estudio en las clases de ciencias, la contrastación de hipótesis, el aprendizaje del manejo de instrumentos, técnicas de laboratorio y de trabajo de campo, la realización de trabajo en grupo, la aplicación de estrategias de investigación, y la comprensión procedimental de la ciencia. Para Tamir (citado por Alonso y Rodríguez ${ }^{18}$ ) el laboratorio se constituye en una herramienta para comprender lo complejo y abstracto de los conceptos científicos, despertar interés, motivación y disfrute por el trabajo de laboratorio; Además, Caamaño ${ }^{19}$ considera que el trabajo de laboratorio contribuye a desarrollar destrezas de comunicación oral y escrita, tales como interpretar instrucciones, planificar investigaciones por escrito, realizar informes, etc. Sin embargo, en investigaciones realizadas sobre el enfoque tradicional de laboratorio, se han revelado pocos beneficios para los estudiantes y sobre la estimación de su potencial didáctico que no ha generado resultados exitosos en el logro de conocimientos, competencias para la comprensión de conceptos científicos, el desarrollo de habilidades técnicas y en el manejo de los procesos de la ciencia (Barbera y Valdez citados por Flores, Caballero y Moreira) ${ }^{20}$. Entre las dificultades que se pueden presentar en la realización de laboratorios, según Hodson ${ }^{21}$ está el que los alumnos realizan un trabajo experimental con materiales reales, pero tienen que realizar la discusión de resultados haciendo uso de conceptos abstractos; además, según Barolli (citada por Baroli et al.) ${ }^{22}$. el trabajo en grupo que se realiza en el laboratorio no es una garantía del éxito del aprendizaje de las ciencias, si la dinámica de grupo no gira en torno de la tarea objetiva.

La enseñanza de algunos conceptos de química no es fácil por lo complejo $\mathrm{y}$ abstracto de lo que en ellos se explica, sin embargo, los recursos o las

16. Aureli Caamaño y Ana Oñorbe, "La enseñanza de la química: conceptos y teorías, dificultades de aprendizaje y replanteamientos curriculares". Alambique: didáctica de las ciencias experimentales, Vol. 41 (2004): 68-81, http://biblioteca.universia.net/html_bura/ficha/params/title/ense\%C3\%B1anzaquimica-conceptos-teorias-dificultades-aprendizaje-replanteamientos-curriculares/id/ 38129531. html

17. D. Hodson, "Hacia un enfoque más crítico del trabajo de laboratorio". Enseñanza de las ciencias, Vol. 12. No. 3, (1994): 299-313, http://www.cneq.unam.mx/programas/actuales/cursos_diplo/cursos/ cursos_SEP/00/primaria/mat_particip_prim/arch_part_prim/S1P1.pdf

18. Carlos Alonso y Geovany Rodríguez. "El laboratorio en la enseñanza de las ciencias naturales: ¿plato fuerte o plato de segunda mesa?". En Educación y Ciencia, Vol. 1 No. 1 (1990): 21-23, http:// educacionyciencia.org/index.php/educacionyciencia/article/view/8/pdf

19. Aureli Caamaño. "Los trabajos prácticos en ciencias". Enseñar Ciencias (Barcelona: Editorial Graó, 2003), 95-118, http://www.cneq.unam.mx/programas/actuales/cursos_diplo/cursos/cursos_ SEP/00/secundaria/mat_particip_secun/02_fisica/arch_partic_fisica/S1P1.pdf

20. Julia Flores, María Caballero y Marco Moreira. "El laboratorio en la enseñanza de las ciencias: una visión integral en este complejo ambiente de aprendizaje". Revista de Investigación, Vol. 33. No. 68, (2009): 75-111, http://www2.scielo.org.ve/pdf/ri/v33n68/art05.pdf

21. D. Hodson, "Hacia un enfoque más crítico del trabajo de laboratorio". Enseñanza de las ciencias, 4.

22. Elisabeth Baroli, Carlos Laburú y Veronica Guridi, "Laboratorio didáctico de ciencias: caminos de investigación". Revista electrónica de enseñanza de las ciencias, Vol. 9. No 1 (2010): 88-100. http:// reec.uvigo.es/volumenes/volumen9/ART6_VOL9_N1.pdf 
herramientas informáticas que ofrecen las TIC como animaciones, aplicativos multimedia, videos, imágenes, documentales, simuladores y tutoriales, puede facilitar esta tarea. Siendo este el motivo por el cual se incluyeron OVAs y un laboratorio virtual de química en el diseño del programa guía de actividades para la enseñanza de la ley de conservación de la masa. Sin embargo, para Cabero $^{23}$ las TIC y su potencial instrumental son solo medios y recursos didácticos que el profesor utiliza de manera adecuada para propiciar ambientes de aprendizaje, lo cual no implica que con ellos se pueda resolver todos los problemas educativos. Según lo propone Cabero, desde la especialidad las TIC son una ayuda en la enseñanza de la química en aspectos como: la utilización de Internet para la presentación y desarrollo de actividades en química; laboratorios virtuales y simuladores; webquest, blogs y wikis en la enseñanza de la química y; las TIC como recursos para la formación del profesorado en la enseñanza de la química.

Un objeto virtual de aprendizaje se define como "una unidad didáctica digital diseñada para alcanzar un objetivo de aprendizaje simple, y para ser reutilizada en diferentes entornos virtuales de enseñanza y aprendizaje, y en distintos contextos de aprendizaje. Debe contar, además, con metadatos que propicien su localización, y permitan abordar su contextualización"24. Mientras que para el Ministerio de Educación Nacional de Colombia "Un objeto de aprendizaje es un conjunto de recursos digitales, autocontenible y reutilizable, con un propósito educativo y constituido por al menos tres componentes internos: contenidos, actividades de aprendizaje y elementos de contextualización. El objeto de aprendizaje debe tener una estructura de información externa (metadatos) que facilite su almacenamiento, identificación y recuperación"25. Por otro lado, los LVQs son herramientas informáticas que simulan un laboratorio de química desde un ambiente virtual, siendo la pantalla el área de trabajo en la cual el estudiante puede manipular equipo de vidrio, equipo de medición, equipo de calentamiento, equipo de filtración, manipulación de reactivos disponibles y realización de diversos procesos, que pueden ser predeterminados o de libre aplicación. Entre las posibles aplicaciones para los LVQs se encuentran; complemento del laboratorio real, instancia de evaluación, reemplazo del laboratorio real y realización de prácticas complejas ${ }^{26}$.

23. Julio Cabero, "Las TICs en la enseñanza de la química: aportaciones desde la Tecnología Educativa". Química: vida y progreso (2007), 1-34, http://tecnologiaedu.us.es/cuestionario/bibliovir/jca16.pdf

24. Gustavo Astudillo, "Análisis del estado del arte de los objetos de aprendizaje. Revisión de su definición y sus posibilidades". Trabajo final especialización Universidad de la Plata (Buenos Aires: Argentina, 2011), http://sedici.unlp.edu.ar/bitstream/handle/10915/4212/all-0001.pdf?sequence=1

25. Objetos virtuales de aprendizaje e informativos. (s.f.), http://www.colombiaaprende.edu.co/html/ directivos/1598/article-172369.html

26. Diego Chiarenza, "Las TICs en la enseñanza de la Química: Laboratorios Virtuales". Tesina en tecnología educativa Universidad Tecnológica Nacional (Buenos Aires: Argentina, 2011), http://issuu.com/ diegochiarenza/docs/tesina_laboratorios_virtuales_de_qu_mica 
Caamaño ${ }^{27}$ plantea que las dificultades en el aprendizaje de los conceptos químicos se ponen de manifiesto en la existencia de un gran número de concepciones alternativas de los estudiantes, que han sido ampliamente estudiadas y que pueden atribuirse a las dificultades intrínsecas y terminológicas de la propia disciplina, al pensamiento y procesos de razonamiento de los estudiantes y al proceso de instrucción recibido. Según Gómez ${ }^{28}$, las ideas y dificultades en el aprendizaje de la química surgen en tres núcleos de contenidos: la naturaleza corpuscular de la materia, la conservación de propiedades no observables y la aplicación cuantitativa de las leyes químicas. Para Pozo y Gómez ${ }^{29}$, una característica habitual de la enseñanza de las ciencias es que los docentes explican o enseñan conceptos, que los alumnos aprenden como una serie de datos que tienen que memorizar o reproducir, que no garantiza comprensión de conceptos, que requieren para su aprendizaje establecer relaciones con los conocimientos previos y poner en marcha procesos cognitivos más complejos. Para que un material de aprendizaje sea comprendido se requiere una organización conceptual interna, de forma que cada elemento tenga conexión lógica o conceptual con otros elementos, que su terminología y vocabulario no sea excesivamente novedoso ni difícil, que tenga en cuenta los conocimientos previos del alumno, su motivación y actitud favorable hacia el aprendizaje; todo esto para alcanzar un aprendizaje significativo que modifique los conocimientos previos y surja un nuevo conocimiento. Aprender química requiere reconocer la existencia de propiedades no observables de la materia que se conservan a pesar los cambios que esta experimente. Cuando la materia experimenta un cambio de estado (cambio físico) la sustancia no cambia su estructura microscópica y conserva su identidad. Por otra parte, en los cambios químicos (reacciones químicas), a partir de las sustancias iniciales (reactivos) se obtienen nuevas sustancias (productos), por lo que no hay conservación de las sustancias pero si conservación de la masa ${ }^{30}$.

En 1789 Lavoisier estableció la ley de conservación de la masa, como una de las leyes fundamentales del cambio químico al afirmar que "Podemos asentar como axioma incontrovertible que, en todas las operaciones del arte y la naturaleza, nada se crea; existe una cantidad igual de materia tanto antes como después del experimento"31. Que ahora gracias a las explicaciones de la teoría atómica la podemos expresar de las siguientes maneras: en una reacción

27. Caamaño y Oñorbe, "La enseñanza de la química: conceptos y teorías, dificultades de aprendizaje y replanteamientos curriculares", 4.

28. Miguel Gómez, "Ideas y dificultades en el aprendizaje de la química". Alambique: didáctica de las ciencias experimentales, No. 7, (1996), 37-44, http://alambique.grao.com/revistas/alambique/007las-ideas-del-alumnado-en-ciencias/ideas-y-dificultades-en-el-aprendizaje-de-la-quimica

29. Pozo y Gómez, Aprender y enseñar ciencia, 2.

30. Pozo y Gómez, 2.

31. Theodore Brown, Eugene Lemay y Bruce Bursten, Química la ciencia central, 7a. edición (México: Prentice Hall Hispanoamericana S.A., 1998). 
química los átomos no son creados ni destruidos por que el mismo número de átomos está presente antes y después de la reacción, o que en una reacción química la masa de los reactivos debe ser igual a la masa de los productos. Para comprender esta importante ley química se requieren comprender conceptos como átomo, molécula, masa molecular, masa molar, cambio físico, cambio químico, ecuación química, balanceo de ecuaciones químicas y se requiere conocer este concepto en temas de estudio posteriores como cálculos estequiométricos entre otros. Así la enseñanza de conceptos en química es una tarea difícil y tiene que transcender de la enseñanza de datos o de hechos, además de superar las ideas previas, preconceptos y preteorias que tienen los estudiantes, que suelen ser difíciles de cambiar e incluso se mantiene luego de la enseñanza.

Por lo previamente expuesto en esta investigación se diseñó y aplicó bajo el enfoque de investigación dirigida un programa guía de actividades que incluye OVAs y el LVQ VLabQ para la enseñanza de la ley de conservación de la masa y se analizó ventajas y desventajas de su aplicación. Se determinaron cambios de actitud mediante un test de Likert y se evaluaron ideas previas y conocimientos sobre la ley de conservación de la masa, comparando resultados antes y después de la realización del programa guía de actividades.

\section{METODOLOGÍA}

La investigación de tipo exploratoria y descriptiva se realizó durante el año escolar 2014, en la I.E.M. Eduardo Romo Rosero del corregimiento de Buesaquillo, municipio de Pasto. La unidad de análisis estuvo constituida por 17 estudiantes de grado décimo, la investigación se realizó durante el cuarto periodo académico entre el 16 de junio y el 28 de noviembre de 2014.

El programa guía de actividades constó de cuatro talleres con actividades de iniciación, desarrollo y finalización; en el taller número cuatro se realizó la práctica de laboratorio virtual. Se determinaron ventajas y desventajas del programa guía de actividades, dedicando una sección aparte a la descripción y evaluación pedagógica del laboratorio virtual $V L a b Q$, teniendo como referencia los criterios para la evaluación pedagógica realizada al laboratorio virtual Model Chemlab, propuestos por Vidal y Gonzáles 32; además de analizar ventajas y desventajas de su aplicación.

Se utilizó una escala de Likert de 30 ítems para la medición de actitudes hacia el estudio de química, utilización de TIC en el aprendizaje, realización de

32. Gonzalo Vidal y Hilda Gonzáles, "Evaluación pedagógica del simulador del laboratorio químico Model Chemlab". Revista Pedagogía Universitaria, Vol. 7. No. 4, (2002), 17-29, http://cvi.mes.edu.cu/ peduniv/base-1/2002-vol.-vii-no.-5/evaluacion-pedagogica-del-simulador-del-laboratorio-quimicomodel-chemlab 
trabajo en grupo, realización de guías de actividades y realización de prácticas de laboratorio. Para la selección y adecuación de ítems se tuvo en cuenta los trabajos de Montoya ${ }^{33}$, Martínez, Villamil y Peña ${ }^{34}$, Molina, Carriazo y Farias ${ }^{35}$ y Astudillo y Chévez ${ }^{36}$, en los que se evaluaron actitudes similares a las de esta investigación. Para las afirmaciones positivas se asignó la siguiente valoración: $\mathrm{TA}=5, \mathrm{~A}=4, \mathrm{I}=3, \mathrm{D}=2$ y $\mathrm{TD}=1$; mientras que para las afirmaciones negativas la escala se invierte, para que un valor cercano a 5 muestre una actitud más positiva. Se promedió el valor obtenido en cada afirmación, y se evaluaron las actitudes con una escala en la que un valor 3 es neutral, valores entre 2-3 representan actitudes negativas, valores entre 3-4 corresponden a actitudes moderadamente positivas y valores entre 4-5 representan actitudes muy positivas $^{37}$.

Para conocer las ideas previas de los estudiantes sobre la ley de la conservación de la masa, se aplicó un test de 3 preguntas de selección múltiple con única respuesta, que incluyó un espacio en blanco para que el estudiante explique o justifique su respuesta. Se realizaron preguntas sobre la combustión de papel en un sistema cerrado ${ }^{38}$, una reacción de precipitación de sulfato de bario y una reacción de formación de dióxido de carbono en un sistema cerrado $^{39}$. En una segunda evaluación se aplicó un test de 13 preguntas de selección múltiple con única respuesta, para evaluar conocimientos específicos en los que se aplica la ley de conservación de la masa; utilizando preguntas liberadas de las pruebas SABER anteriores al año 2014.

\section{RESULTADOS Y ANÁLISIS}

\subsection{Programa guía de actividades}

Los talleres del programa guía de actividades se diseñaron teniendo en cuenta los estándares básicos de competencias, desempeños esperados, una

33. Diana Montoya, "Diseño e implementación de guías para el aprendizaje de la materia y sus propiedades apoyadas en herramientas virtuales". Trabajo de grado maestría Universidad Nacional de Colombia, 2012, http://www.bdigital.unal.edu.co/9100/1/8410503.2012.pdf

34. Leonardo Martínez, Yenny Villamil y Diana Peña, "Actitudes favorables hacia la química a partir del enfoque Ciencia, Tecnología, Sociedad y Ambiente (CTSA)". I Congreso Iberoamericano de Ciencia, Tecnología e Innovación CTS+I. (Junio, 2006), http://www.oei.es/memoriasctsi/mesa4/m04p53b.pdf

35. Manuel Molina, José Carriazo y Diana Farías, "Actitudes hacia la química de estudiantes de diferentes carreras universitarias en Colombia". Quim. Nova, Vol. 34. No. 9, (2011), 1672-1677, http://www. scielo.br/pdf/qn/v34n9/v34n9a32.pdf

36. Marta Astudillo y Florlenis Chévez, "Valoración de las actitudes los estudiantes frente al uso de TIC". Revista Digital de la Universidad Autónoma de Chiapas. (s. f.), http://espacioimasd.unach.mx/ articulos/num2/pdf/valoracion_de_las\%20actitudes_de_estudiantes_universitarios_frente_al_uso_ de_las_TIC.pdf

37. Molina, Carriazo y Farías, "Actitudes hacia la química de estudiantes de diferentes carreras universitarias en Colombia", 8.

38. Oñorbe y Sánchez, "La masa no se crea ni se destruye. ¿Estáis seguros?", 1

39. Curtis Pyke y Rob Ochsendorf. Conservation of Matter Assessment Manual. Scale-uP Research Staff, (2004). http://www2.gwu.edu/ scale-up/documents/COMAManual.pdf 
secuencia adecuada de actividades y contenidos, selección de OVAs que ayuden a explicar y comprender los conceptos de química estudiado y sobre todo brinden la posibilidad la alumno de participar activamente en su aprendizaje. La estructura general del programa guía de actividades comprende actividades de iniciación: motivación inicial, determinación de conocimientos previos, generación de un conflicto cognitivo y relación con la historia de la química; actividades de desarrollo: introducción a conceptos científicos, ejercicios de aplicación de conceptos científicos, actividades relacionadas con la investigación y relación con la vida cotidiana; y actividades de finalización: elaboración de síntesis y evaluación del aprendizaje.

El programa guía de actividades constó de cuatro talleres que desarrollan los siguientes temas:

Tabla 1. Talleres programa guía de actividades

\begin{tabular}{|c|l|c|}
\hline TALLER & \multicolumn{1}{|c|}{ TEMAS } & $\begin{array}{c}\text { Número de } \\
\text { actividades }\end{array}$ \\
\hline 1 & Molécula, masa molecular, masa molar y mol. & 18 \\
\hline 2 & Cambios físicos, cambios químicos y ecuaciones químicas & 15 \\
\hline 3 & $\begin{array}{l}\text { Ley de conservación de la masa, balanceo de ecuaciones y } \\
\text { cálculos químicos }\end{array}$ & 19 \\
\hline 4 & $\begin{array}{l}\text { Ley de conservación de la masa - Laboratorio virtual de química } \\
\text { (VLabQ). }\end{array}$ & 10 \\
\hline \multicolumn{2}{|c|}{ 45\% de las actividades incluyeron OVAS. } \\
\hline
\end{tabular}

Fuente: Esta investigación

Uno de los aspectos más importantes del programa guía de actividades es la utilización de OVAs para la enseñanza de conceptos de química necesarios para comprender la ley de conservación de la masa, estos recursos digitales se seleccionaron con un propósito educativo mediante el buscador Google, teniendo en cuenta su libre acceso, calidad en la presentación gráfica, desarrollo correcto de teorías, leyes y conceptos de química e interactividad con el alumno. Entre los recursos informáticos que se utilizados tenemos: lectura de documentos en línea, consultas en Wikipedia, videos para explicar conceptos, videos introductorios a ciertos temas, videos de reacción químicas, visor de moléculas 3D, una aplicación para determinar masa molar y masa molecular, tutoriales de algunos temas estudiados en el programa guía de actividades, evaluaciones en línea, simulaciones y ejercicios de balanceo de ecuaciones químicas.

Entre las ventajas de la aplicación de programa guía de actividades se encontró que: el uso pedagógico de TIC aumento el interés y la motivación aprender química, mejoró su participación en el trabajo en grupo y el 
estudiante asume un papel activo en su aprendizaje. Exigió al docente ser competente en el uso de las TIC y utilizar pedagógicamente estos recursos, además de ser un dinamizador del proceso de aprendizaje. Una características importantes del programa guía fue fomentar un aprendizaje significativo y proporcionar condiciones para una evaluación permanente y para aprender. Como desventajas se pueden enunciar las siguientes: se presentaron diferentes ritmos de trabajo en los estudiantes, algunas de las actividades resultaron ser muy largas y extensas, por carecer de un laboratorio de química no se realizó una investigación experimental del tema estudiado, es una metodología adecuada para grupos pequeños de estudiantes, y limitó el desarrollo de contenidos por lo cual fueron seleccionadas temáticas específicas que se consideró necesarias para la enseñanza de la ley de conservación de la masa. Algunas de las desventajas enunciadas coinciden con las dificultades o inconvenientes de la aplicación de los programas guía de actividades reportadas por Gil y Martínez ${ }^{40}$ y Hierrezuelo et al. ${ }^{41}$.

\subsection{Taller 4 del programa guía de actividades: ley de conservación de la masa - laboratorio virtual VLabQ}

Se dedica una sección aparte al taller 4 del programa guía de actividades porque en él se incluyó la realización de práctica de laboratorio virtual sobre la ley de conservación de la masa, utilizando el laboratorio virtual VLabQ. A continuación se describen algunas características el laboratorio virtual, un resumen de las actividades del taller 4 y se finaliza con la evaluación pedagógica realizada a VLabQ.

\subsubsection{Características generales laboratorio virtual VLabQ y programa guía}

El laboratorio virtual de química VLabQ de Sibees Soft, ofrece una versión gratuita (Freeware) en idioma español que contiene 5 prácticas de laboratorio sobre: conservación de la materia, destilación simple, reversibilidad de las reacciones, titulación ácido base y calor específico. En el menú principal, en la etiqueta equipo, se despliegan todas las opciones de equipos y materiales con los que cuenta el laboratorio virtual (Figura 1): vasos de precipitados, matraces Erlenmeyer, matraz bola, reactor, buretas, probetas, pipetas, tubo de ensayo, equipo de medición (pHmetro, termómetro, conductímetro y balanza), equipo térmico (mechero Bunsen, parrilla y baño de hielo), agitador de vidrio, vidrio de reloj, cápsula de porcelana y calorímetro.

40. Gil y Martínez, "Los programas-guía de actividades: una concreción del modelo constructivista de aprendizaje de las ciencias", 3 .

41. Hierrezuelo, Molina y Yus, "Una nueva generación de materiales curriculares para la enseñanza de la ciencia: los programas-guía de actividades", 4. 
Figura 1. Materiales y equipos disponibles en el laboratorio virtual VLabQ

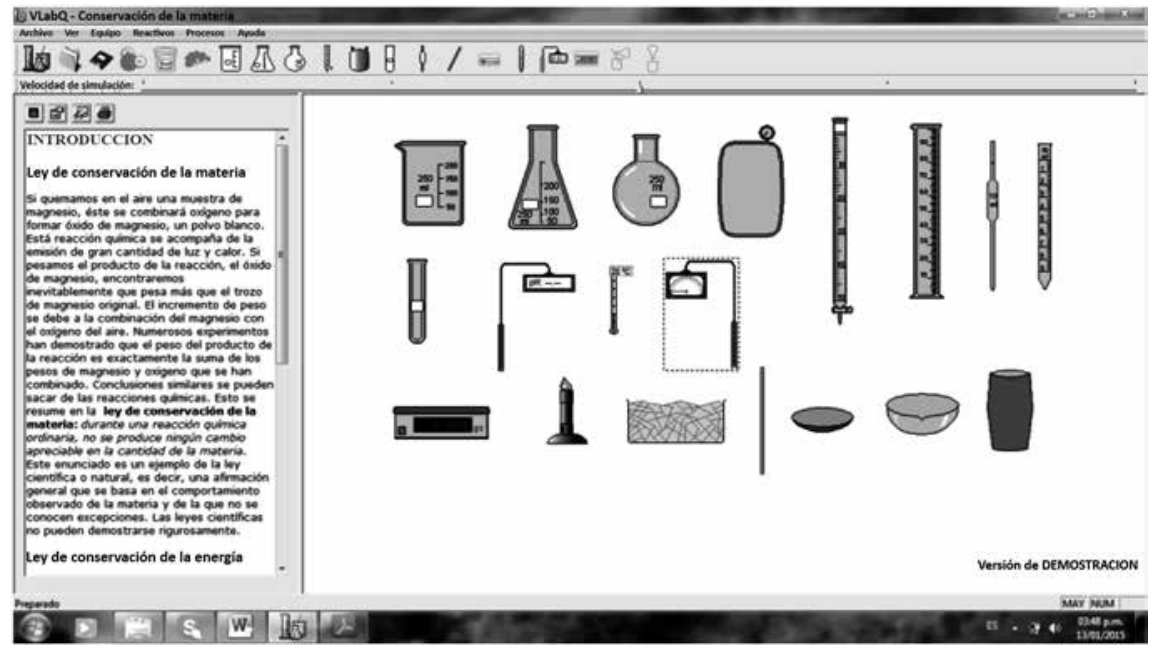

Fuente: Laboratorio virtual VLabQ

Una vez se selecciona la práctica de laboratorio a realizar, en la ventana información de prácticas se activa las pestañas de introducción, procedimiento, resultados y análisis. En la introducción se presentan aspectos teóricos básicos relacionados con la práctica; en procedimiento se describe paso a paso la utilización de materiales y reactivos; en resultados y análisis se orienta la toma de datos, se presentan preguntas para tener en cuenta en el análisis de resultados y se siguiere la búsqueda de ecuaciones químicas.

Como las prácticas son preestablecidas, el alumno tiene que realizar el procedimiento descrito paso a paso en la mesa de trabajo. Los materiales y equipos para la realización de la práctica se obtienen seleccionándolos de la etiqueta equipos del menú principal, como también, dando clic en el icono que representa el material de laboratorio en la barra de herramientas. El material que se selecciona se ubica directamente en la mesa de trabajo. Las operaciones básicas de laboratorio que se pueden realizar en la versión libre del laboratorio virtual VLabQ son las siguientes: Medir masa, volumen, temperatura, $\mathrm{pH}$ y conductividad. Calentamiento con mechero Bunsen o parrilla. Enfriamiento en baño de hielo. Procesos como decantar y transferir. Filtrar a través de un embudo Buchner. Agitar con varilla de vidrio.

En la presente investigación se utilizó el laboratorio virtual VLabQ para realizar la práctica de laboratorio sobre conservación de la masa, que simula las siguientes reacciones: 


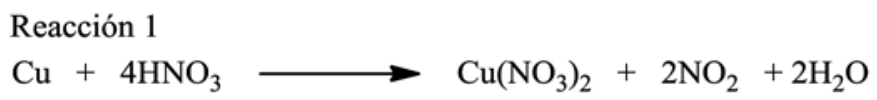

Reacción 2

$\mathrm{Cu}\left(\mathrm{NO}_{3}\right)_{2}+2 \mathrm{NaOH} \longrightarrow \mathrm{Cu}(\mathrm{OH})_{2}+2 \mathrm{NaNO}_{3}$

Reacción 3

$\mathrm{Cu}(\mathrm{OH})_{2}+\mathrm{H}_{2} \mathrm{SO}_{4} \longrightarrow \mathrm{CuSO}_{4}+2 \mathrm{H}_{2} \mathrm{O}$

Reacción 4

$\mathrm{CuSO}_{4}+\mathrm{Zn} \longrightarrow \mathrm{Cu}+\mathrm{ZnSO}_{4}$

Proceso químico en el cual se inicia con 2 gramos de cobre en la reacción 1 y se finaliza con 2 gramos de cobre en la reacción 4 .

\section{Programa guía de actividades práctica de laboratorio virtual}

En las actividades del programa guía se desarrollan los siguientes aspectos: video sobre el laboratorio de química, operación del laboratorio virtual, conocimiento de materiales y equipo, consulta de uso y función del materiales y equipos, realización de operaciones básicas de laboratorio, conocimiento de normas de bioseguridad, formulación de un proyecto de investigación basado en la práctica de laboratorio virtual, realización del laboratorio virtual sobre conservación de la masa, observación de videos reales de las reacciones químicas simuladas. Para el análisis de resultados, se consultó en Internet las ecuaciones que explican las reacciones químicas observadas, se realizó cálculos estequiométricos y se analizaron los resultados teniendo en cuenta la hipótesis y los objetivos con base en los aspectos teóricos aplicados en la práctica.

\section{Evaluación Pedagógica del laboratorio virtual VLabQ}

La versión libre del laboratorio virtual VLabQ contiene cinco prácticas de laboratorio prediseñadas que no se pueden modificar, no se plantean situaciones problema que generen interés y motiven el aprendizaje, porque está diseñado como una práctica de laboratorio tradicional tipo receta de cocina. Presenta información teórica resumida de la práctica a realizar, se indica el procedimiento y se dan algunas indicaciones para la toma de datos y análisis de resultados. Por su diseño el laboratorio virtual no promueve la emisión de hipótesis. En el laboratorio virtual no se pueden incluir recursos multimedia adicionales que complementen o profundicen en los temas estudiados. El software de VlabQ no tiene herramientas para facilitar la interacción, comunicación y retroalimentación en el proceso de aprendizaje 
entre el estudiante y el docente; además, no cuenta con herramientas de control de aprendizaje y realización de actividades de síntesis. Una de las ventajas del laboratorio virtual es que se puede realizar cuantas veces se quiera.

Como el propósito de realizar el laboratorio virtual era acercar al estudiante a la experimentación en un laboratorio de química, en el programa guía de actividades se incluyeron actividades complementarias a la sola realización de la práctica virtual sobre la ley conservación de la masa. Es así como el estudiante propuso un proyecto semejante a un proyecto de investigación, identificando un problema a estudiar, redactó una justificación, formuló hipótesis y objetivos, se buscó que comprenda la metodología, analice los resultados con base en conocimientos aprendidos y este en capacidad de realizar un informe de laboratorio. Al tener en cuenta que el laboratorio virtual VLabQ no incluye actividades para complementar o reforzar los temas estudiados, en el programa guía se incluyeron videos, búsqueda de información e imágenes y realización de consultas. Durante la realización de la práctica de laboratorio virtual se generó un ambiente que facilitó la comunicación entre docente y estudiante, que además permitió la realización de un proceso de evaluación permanente y para el aprendizaje, no contemplado en el software del laboratorio virtual. La posibilidad de repetir la práctica de laboratorio cuantas veces sea necesario, facilitó la retroalimentación que se realizó para la comprensión y el aprendizaje de la ley de conservación de la masa.

En la evaluación pedagógica realizada a VLabQ, se coincide con lo expuesto por Chiarenza ${ }^{42}$ sobre este mismo laboratorio virtual en los siguientes aspectos: la organización del laboratorio virtual es aceptable, no tiene secuencia de contenidos solo se incluye una lista de prácticas, las prácticas de laboratorio tienen una profundidad y complejidad baja, es adecuado para procesos presenciales y semipresenciales, su carácter autodidacta es bajo al no incluir actividades de refuerzo, ampliación, profundización y autoevaluación. También se encuentran similitudes de la evaluación pedagógica de VLabQ con la evaluación pedagógica de Model Chemlab ${ }^{43}$ en que su formato se asemeja al de una práctica de laboratorio tradicional, en la que se presenta la introducción, el procedimiento a realizar y los datos que se deben tomar y sirve para comprobar a través de la práctica virtual algunos de los temas estudiados teóricamente.

\subsubsection{Ventajas y desventajas laboratorio virtual VLabQ}

Entre las ventajas de la realización del laboratorio virtual se identificaron las siguientes: proporciona un ambiente de enseñanza protegido y seguro, se realizó una práctica que sería imposible de realizar si no se tiene un laboratorio de química, acerca a los estudiantes a lo es la investigación y el trabajo en

42. Chiarenza, "Las TICs en la enseñanza de la Química: Laboratorios Virtuales", 6

43. Vidal y Gonzáles, "Evaluación pedagógica del simulador del laboratorio químico Model Chemlab", 7. 
un laboratorio de química, al motivación e interés en los estudiantes ayuda a la comprensión de conceptos científicos, permite la realización de trabajo individual y grupal, las prácticas se realizan en un tiempo corto, se introduce las TIC al plan de estudio de grado décimo y se puede adaptar a diversas propuestas pedagógicas. Ventajas que se describen también por Cataldi, Chiarenza, Dominighini, y Lage ${ }^{44}$ en el trabajo denominado clasificación de laboratorios virtuales de química y propuesta de evaluación heurística. Como desventajas de la realización de la práctica de laboratorio virtual se encontró que no proporciona una experiencia real del trabajo en el laboratorio, no se desarrollan habilidades para el manejo de materiales y equipo de laboratorio, se genera una falsa seguridad en la manipulación de reactivos químicos y su interacción, motiva el interés de los estudiantes pero no se puede comparar con la realización del laboratorio real y debe considerarse una herramienta complementaria para la enseñanza de la química ${ }^{45}$.

\subsection{Test de Likert para determinar cambios de actitud en los estudiantes}

Cada tabla contiene información sobre el número de Ítem que corresponde a cada afirmación y el valor promedio obtenido en el pretest y postest, luego se realiza el análisis correspondiente al cambio o no de actitud presentado en los estudiantes.

\section{Actitud hacia el estudio de química}

Tabla 2. Actitud hacia el estudio de química

\begin{tabular}{|c|l|c|c|}
\hline ÍTEM & \multicolumn{1}{|c|}{ AFIRMACIÓN } & PRETEST & POSTEST \\
\hline 1 & Me gustan las clases de química porque son interesantes & 4,4 & 4,3 \\
\hline 6 & Me agrada más la clase de química que otras asignaturas & 2,9 & 3,4 \\
\hline 11 & Me gusta estudiar y aprender química & 3,6 & 4,0 \\
\hline 16 & Tengo dificultades en el aprendizaje de química & 2,9 & 3,7 \\
\hline 21 & Los conceptos que se estudian en química son difíciles de aprender & 2,7 & 3,7 \\
\hline 25 & Lo que aprendo en química es de utilidad para mi formación personal & 3,6 & 4,3 \\
\hline 28 & $\begin{array}{l}\text { Las clases de química me agradan porque encuentro relación con la } \\
\text { vida cotidiana }\end{array}$ & 3,8 & 3,9 \\
\hline 29 & $\begin{array}{l}\text { Reconozco aplicaciones de química que ha mejorado nuestra calidad } \\
\text { de vida }\end{array}$ & 3,2 & 4,0 \\
\hline 30 & Lo que aprendo en química solo sirve para pasar la asignatura & 4,2 & 4,3 \\
\hline
\end{tabular}

Fuente: Esta investigación

44. Zulma Cataldi, Diego Chiarenza, Claudio Dominighini, y Fernando Large, “Clasificación de laboratorios virtuales de química y propuesta de evaluación heurística. Tecnología informática aplicada a la educación". Conferencia, XIII Workshop de Investigadores en Ciencias de la Computación (Argentina: Universidad Nacional del Rosario, 2011), http://hdl.handle.net/10915/19937

45. Pedro Martínez, "Química de Bachillerato y laboratorios virtuales". II jornadas sobre la enseñanza de las ciencias e ingenierías, (s.f.) http://www.murciencia.com/upload/comunicaciones/37_quimica_ laboratorios_virtuales.pdf 
Tanto en el pretest como en el postest se observan actitudes muy positivas en el ítem 1 gusto e interés hacia las clases de química, y en el ítem 30, que muestran que los estudiantes no están de acuerdo que lo que se aprende solo sirve para pasar la materia. En el ítem 25 concerniente al aprendizaje de química y su utilidad para la formación personal, se presentó un cambio de actitud de moderadamente positiva (pretest) a muy positiva (postest). Se presentan actitudes moderadamente positivas en el pretest y postest en los ítems 11, 28 y 29 que evaluaron actitudes hacia estudiar y aprender química, la utilidad de lo que se aprende, y los beneficios de la química en la vida cotidiana.

En el pretest se presentaron actitudes negativas en el ítem 6, que evalúa el agrado de estudiar química comparada con otras asignaturas; ítem 16 según el cual los estudiantes consideran que tienen dificultades en aprender química y el ítem 21 que revela que el aprendizaje de conceptos de química no es fácil para los estudiantes. Sin embargo, en el postest estas actitudes negativas (ítems 6,16 y 21) cambiaron a actitudes moderadamente positivas.

Luego de realizar el programa guía de actividades las actitudes hacia el estudio de química, mejoraron ubicándose entre moderadamente positivas y muy positivas. Este cambio de actitud en los estudiantes se puede atribuir, a que en el diseño del programa guía de actividades se relacionó adecuadamente los contenidos de aprendizaje, se plantearon actividades para conocer las ideas previas de los estudiantes, se realizó una enseñanza no tradicional donde el estudiante asume una posición activa y el docente orienta el proceso de aprendizaje, la utilización de OVAs generó ambientes para alcanzar un aprendizaje significativo y se utilizó la evaluación como una estrategia más para el aprender.

\section{Actitud hacia la utilización de TIC en procesos de aprendizaje}

Tabla 3. Actitud hacia la utilización de TIC en procesos de aprendizaje

\begin{tabular}{|c|l|c|c|}
\hline ÍTEM & \multicolumn{1}{|c|}{ AFIRMACIÓN } & PRETEST & POSTEST \\
\hline 2 & $\begin{array}{l}\text { Utilizo las tecnologías de la información y la comunicación (TIC) para } \\
\text { estudiar }\end{array}$ & 2,5 & 4,7 \\
\hline 7 & Empleo mis habilidades en el uso de Internet para aprender química & 2,2 & 4,6 \\
\hline 12 & $\begin{array}{l}\text { Internet y las herramientas virtuales facilitan y ayudan a un mejor } \\
\text { aprendizaje }\end{array}$ & 2,8 & 4,5 \\
\hline 17 & Me gustan las clases en las que se aplican TIC para enseñar y aprender & 3,9 & 4,5 \\
\hline 22 & Las TIC aplicadas a la enseñanza ayudan al aprendizaje de química & 3,9 & 4,3 \\
\hline 26 & Me gusta utilizar herramientas virtuales para aprender química & 3,1 & 4,6 \\
\hline
\end{tabular}

Fuente: Esta investigación 
En la tabla 3 se observa que en el pretest los estudiantes presentaron una actitud modernamente positiva, en el gusto hacia las clases en las se aplican las TIC (ítem 17), como también, en considerar que las herramientas virtuales ayudaría al aprendizaje de química (ítem 22) y que les gusta estudiar química utilizando herramientas virtuales (ítem 26). Esta actitud moderadamente positiva en el pretest, se puede interpretar desde la expectativa que tenían los estudiantes hacia la utilización de las TIC para aprender. Luego de realizar el programa guía de actividades las actitudes moderadamente positivas del pretest en los ítems 17, 22 y 26 cambiaron a actitudes muy positivas en el postest.

En el pretest se presentaron actitudes negativas hacia la utilización efectiva de las TIC para estudiar y el uso de habilidades de Internet para aprender química (ítem 2 y 7), y en que los estudiantes están en desacuerdo con la afirmación que las TIC facilitan y ayudan a un mejor aprendizaje (ítem 12). Se considera que estas actitudes fueron negativas porque hasta ese momento el docente no había utilizado las TIC para enseñar y los estudiantes no habían utilizado estos recursos tecnológicos para aprender química. Luego de realizar el programa guía de actividades, las actitudes evaluadas en los ítems 2, 7 y 12 cambiaron a muy positivas. Este cambio favorable en la actitud de los estudiantes se puede deber a que en el programa guía se realizó uso pedagógico de las TIC, incluyendo actividades en las cuales se utilizó Internet como fuente de información, se usó OVAs para la enseñanza y aprendizaje de conceptos de química, se emplearon simuladores, animaciones, aplicativos multimedia y un laboratorio virtual de química. Además de videos para la introducción o explicación de algunos temas de estudio y realización de talleres y evaluaciones virtuales.

Teniendo en cuenta el cambio de actitud que presentaron los estudiantes hacia el uso de las TIC para aprender y las ventajas de utilizar OVAs en un proceso de enseñanza y aprendizaje enunciadas por Jurado et $a l .{ }^{46}$ se considera que en esta investigación el uso de OVAs generó participación y dinamizó el proceso de aprendizaje, se propició espacios para aprendizajes significativos, se requirió que el docente sea competente en el uso pedagógico de las TIC y que los estudiantes aprendieran a aprender mediante los OVAs.

\section{Actitud hacia la realización de trabajo en grupo}

Con relación a la realización de trabajo en grupo, la actitud de los estudiantes en los ítems 3, 18 y 23 en el pretest y postest se valoró con promedio superior

46. Gloria Jurado, María Rosero y Betty Velasco, "Objetos virtuales de aprendizaje (OVA) como mediadores en el proceso de aprendizaje de funciones trigonométricas" (Trabajo de grado maestría Universidad de Nariño, Pasto, Colombia, 2010). 
a 4 (actitud muy positiva), indicando que en el trabajo en grupo se aprende mejor química (ítem 3), se interesan más por aprender esta ciencia (ítem 18), y al considerar que el trabajo en grupo mejora la integración y el apoyo entre compañeros (ítem 23). Estos resultados se pueden explicar, al tener en cuenta que la realización de trabajo en grupo mediante "guías de aprendizaje" ha sido una de las actividades utilizada para la enseñanza y aprendizaje de química en la IEM Eduardo Romo Rosero del Corregimiento de Buesaquillo desde el año 2010 y es el núcleo de la realización del programa guía de actividades.

Tabla 4. Actitud hacia la realización de trabajo en grupo

\begin{tabular}{|c|l|c|c|}
\hline ÍTEM & \multicolumn{1}{|c|}{ AFIRMACIÓN } & PRETEST & POSTEST \\
\hline 3 & $\begin{array}{l}\text { Se aprende mejor química cuando se realizan actividades de trabajo } \\
\text { en grupo }\end{array}$ & 4,2 & 4,2 \\
\hline 8 & $\begin{array}{l}\text { Me gusta el trabajo en grupo porque participo en la realización de } \\
\text { actividades }\end{array}$ & 3,6 & 4,3 \\
\hline 13 & $\begin{array}{l}\text { Prefiero que el profesor se dedique solo a explicar los temas de } \\
\text { química durante las clases }\end{array}$ & 3,5 & 3,8 \\
\hline 18 & Me intereso por aprender química cuando se realiza trabajo en grupo & 4,4 & 4,3 \\
\hline 23 & El trabajo en grupo mejora la integración yel apoyo entre compañeros & 4,4 & 4,5 \\
\hline
\end{tabular}

Fuente: Esta investigación

Para el ítem 8 que evalúa la disposición de los estudiantes a participar en la realización de actividades de aprendizaje en grupo se presenta un cambio de actitud de moderadamente positiva en el pretest a muy positiva en el postest. Los resultados del pretest y postest para el ítem 13, indican una actitud moderadamente positiva hacia la afirmación sobre la preferencia hacia la realización de clases tradicionales.

El trabajo en grupo es una de las estrategias que se utilizó para desarrollar el enfoque de enseñanza de las ciencias por investigación dirigida, porque permite abordar situaciones problemáticas desde una orientación científica y profundizar en su estudio ${ }^{47}$; que entre sus ventajas presenta el trabajo colectivo en pequeños grupos que incrementa la participación, la creatividad y el intercambio de información ${ }^{48}$.

\section{Actitud hacia el desarrollo guías de actividades}

En los ítems 4, 9, 14 y 19 se evaluó la actitud de los estudiantes hacia el desarrollo de guías de actividades. Los resultados del pretest y postest indican que para el ítem 14 la actitud de los estudiantes es muy positiva,

47. Campanario y Moya, “¿Cómo enseñar ciencias? Principales tendencias y propuestas”, 3.

48. Gil, "Contribución de la historia y de la filosofía de las ciencias al desarrollo de un modelo de enseñanza/aprendizaje como investigación", 2. 
porque consideran que la realización de guías de actividades facilita el aprendizaje de química. Mejoró la actitud hacia poder realizar las actividades planteadas en una guía de aprendizaje (ítem 9), que paso de moderadamente positiva en el pretest a muy positiva en el postest. Otro cambio de actitud se presentó en el ítem 19, que cambió de una actitud negativa en el pretest a moderadamente positiva en el postest, evidencia de que los estudiantes no están completamente de acuerdo con esta afirmación y que les gusta realizar las guías de actividades. La valoración del ítem 4 con promedios de 3,5 y 3,8 en el pretest y postest respectivamente, indica que los estudiantes no están completamente de acuerdo con esta afirmación, por lo que se valoró la actitud como moderadamente positiva.

Tabla 5. Actitud hacia desarrollo guías de actividades

\begin{tabular}{|c|l|c|c|}
\hline ÍTEM & \multicolumn{1}{|c|}{ AFIRMACIÓN } & PRETEST & POSTEST \\
\hline 4 & El estudio de química es más difícil siguiendo guías de actividades & 3,5 & 3,8 \\
\hline 9 & Puedo realizar las actividades planteadas en una guía de aprendizaje & 3,7 & 4,2 \\
\hline 14 & Las guías de actividades facilitan el aprendizaje de química & 4,4 & 4,3 \\
\hline 19 & $\begin{array}{l}\text { Prefiero la explicación del profesor antes que realizar guías de } \\
\text { actividades }\end{array}$ & 2,7 & 3,2 \\
\hline
\end{tabular}

Fuente: Esta investigación

Las actitudes de los estudiantes hacia la realización de guías de actividades, en el postest se encuentran entre moderadamente positivas y muy positivas, resultados que se pueden atribuir a la metodología activa del programa guía de actividades, que permite al alumno construir conocimiento y la interacción entre compañeros gracias al trabajo en grupo; que para Hierrezuelo, Molina y Yus $^{49}$ son algunas de las ventajas de esta estrategia didáctica. Además, hay que tener en cuenta que la realización del programa guía de actividades incrementó la participación y la motivación de los estudiantes, que son según Campanario y Moya ${ }^{50}$ son otras de las ventajas de la realización de los programas guía y que se consiguen gracias al trabajo de un docente que orienta el desarrollo de las actividades, explica y resuelve dudas, complementa el trabajo de los grupos y coordinar la socialización de actividades.

\section{Actitud hacia la realización de prácticas de laboratorio de química}

Para la interpretación de los resultados del test de Likert sobre la actitud hacia la realización de prácticas de laboratorio, es necesario tener en cuenta que la IEM Eduardo Romo Rosero no cuenta con laboratorio de química,

49. Hierrezuelo, Molina y Yus, "Una nueva generación de materiales curriculares para la enseñanza de la ciencia: los programas-guía de actividades", 4.

50. Campanario y Moya, “Cómo enseñar ciencias? Principales tendencias y propuestas”, 3. 
motivo por el cual los estudiantes no han tenido la experiencia de realizar trabajo de laboratorio y mucho menos conocer materiales, equipos y reactivos químicos; solo se han realizado algunas experiencias de laboratorio utilizando materiales y reactivos que se pueden encontrar en la cotidianidad. Por lo tanto, se considera que algunas de las actitudes valoradas en el pretest reflejan las ideas que tienen los estudiantes sobre el trabajo en un laboratorio de química y sus expectativas hacia la realización de prácticas de laboratorio. De ahí la importancia de comparar mediante el test de Likert las actitudes de los estudiantes antes y después de realizar un laboratorio virtual de química.

Tabla 6. Actitud hacia la realización de prácticas de laboratorio de química

\begin{tabular}{|c|c|c|c|}
\hline ÍTEM & AFIRMACIÓN & PRETEST & POSTEST \\
\hline 5 & $\begin{array}{l}\text { La realización de prácticas de laboratorio motiva el estudio de } \\
\text { química }\end{array}$ & 3,3 & 4,3 \\
\hline 10 & $\begin{array}{l}\text { La realización de prácticas de laboratorio ayuda al aprendizaje de } \\
\text { química }\end{array}$ & 4,0 & 4,5 \\
\hline 15 & $\begin{array}{l}\text { Lo que aprendo en química me permite explicar lo observado en las } \\
\text { prácticas de laboratorio }\end{array}$ & 2,5 & 4,3 \\
\hline 20 & $\begin{array}{l}\text { Me gusta realizar experimentos de química porque aprendo a } \\
\text { investigar }\end{array}$ & 4,2 & 4,4 \\
\hline 24 & Valoro y respeto las normas de trabajo en el laboratorio de química & 2,7 & 4,4 \\
\hline 27 & $\begin{array}{l}\text { Conozco algunos de los procedimientos de trabajo que se realizan en } \\
\text { un laboratorio de química }\end{array}$ & 1,7 & 4,1 \\
\hline
\end{tabular}

Fuente: Esta investigación

En el pretest y postest los estudiantes presentan una actitud muy positiva hacia la realización de experimentos de química (ítem 20). La actitud de los estudiantes cambio de moderadamente positiva a muy positiva en lo relacionado a la realización de prácticas de laboratorio y como estas motivan el estudio (ítem 5) y ayudan al aprendizaje de química (ítem 10). El cambio más destacado de actitudes negativas en el pretest a actitudes muy positivas en el postest, se presentó en los ítems 15, 24 y 27; el primero porque los estudiantes consideran que lo que se aprende en química les permitió explicar lo realizado en el práctica de laboratorio; el segundo por el conocimiento de normas básicas de bioseguridad para el trabajo de laboratorio y el tercero porque los estudiantes conocieron algunos procedimientos que se realizan en un laboratorio de química.

El acercamiento de los estudiantes al trabajo que se realiza en un laboratorio de química se consiguió en el cuarto taller del programa guía de actividades, en el cual se aprende a utilizar el laboratorio virtual VLabQ y se realiza una práctica virtual sobre la ley de conservación de la masa. En este programa guía se realizaron las siguientes actividades: estudio de un video 
sobre lo que es un laboratorio de química; conocimiento y usos del material de laboratorio de disponible en VLabQ; búsqueda de información sobre pictogramas de seguridad, manejo de reactivos y consulta de normas de bioseguridad y realización de procedimientos propios de un laboratorio. Sin embargo, con la sola realización del laboratorio virtual no se alcanzan en su totalidad los objetivos que se buscan con la realización de trabajos prácticos en la enseñanza de la ciencia, como el aprendizaje del manejo de instrumentos y técnicas de laboratorio, la aplicación básica de estrategias para la resolución de problemas y la comprensión básica de los procedimientos de la ciencia ${ }^{51}$.

\subsection{Test para conocer ideas previas sobre la ley de conservación de la masa}

Los resultados del pretest y postest se presentan en una tabla que contiene el enunciado, las preguntas realizadas, las opciones de respuesta y los porcentajes obtenidos para cada opción de respuesta. Luego, se comparan y analizan los resultados teniendo en cuenta las generalidades encontradas.

\section{Pregunta 1. Combustión de papel en un sistema cerrado}

Tabla 7. Pregunta y respuestas combustión de papel en un sistema cerrado.

\begin{tabular}{|c|c|c|c|c|}
\hline \multirow{2}{*}{ ENUNCIADO } & \multirow{2}{*}{ PREGUNTA } & \multirow{2}{*}{$\begin{array}{l}\text { OPCIÓN DE } \\
\text { RESPUESTA }\end{array}$} & PRETEST & POSTEST \\
\hline & & & $\%$ & $\%$ \\
\hline \multirow{5}{*}{$\begin{array}{l}\text { En una botella, como la de } \\
\text { la figura, situada sobre una } \\
\text { balanza, se introduce un papel } \\
\text { encendido que pesa } 20 \text { gramos } \\
\text { y se cierra inmediatamente. } \\
\text { Una vez cerrada, la aguja de } \\
\text { la balanza marca } 520 \text { gramos } \\
\text { (Oñorbe \& Sánchez, } 1992 \text { ). }\end{array}$} & \multirow{5}{*}{$\begin{array}{l}\text { Cuando el papel se } \\
\text { queme totalmente la } \\
\text { balanza marcará... }\end{array}$} & Menos de $500 \mathrm{~g}$ & 23,53 & 5,88 \\
\hline & & $500 \mathrm{~g}$ & 47,06 & 0,00 \\
\hline & & Entre 500 y $520 \mathrm{~g}$ & 11,76 & 5,88 \\
\hline & & $520 \mathrm{~g}$ & 11,76 & 82,35 \\
\hline & & Más de $520 \mathrm{~g}$ & 5,88 & 5,88 \\
\hline
\end{tabular}

Fuente: Esta investigación

En el pretest solo el 11,76\% de los estudiantes escogió la respuesta correcta, que la balanza marcara 520 g luego de la combustión, porque la reacción se realiza en un sistema cerrado; en el postest este porcentaje aumentó a 82,35\%. En el pretest la explicación generalizada fue que la masa se mantiene igual, mientras que en el postest las explicaciones dadas por los estudiantes fueron: se tiene que cumplir la ley de conservación de la masa; la masa es la misma solo cambia la forma, las cenizas y el humo pesan lo mismo; la materia solo se transformó, pesa lo mismo porque el humo no puede salir; las sustancias

51. Caamaño, "Los trabajos prácticos en ciencias", 4. 
cambiaron de estado pero pesan lo mismo; lo que queda de la combustión pesa lo mismo; al ser el sistema cerrado pesa lo mismo; cambia la masa pero el peso sigue igual.

En el caso de las respuestas incorrectas, en el pretest se presentaron los siguientes resultados: para el 23,53\% de estudiantes la masa es menor de 500 $\mathrm{g}$, porque al quemarse se consume aire y el papel pierde peso al convertirse en cenizas; que el papel se deshace y en la botella queda humo y que las cenizas son menos densas que el papel. Por otro lado, el $47,06 \%$ consideró que la masa luego de la combustión será de $500 \mathrm{~g}$, porque al quemarse el papel se pierde $20 \mathrm{~g}$ y quedan $500 \mathrm{~g}$ correspondientes a la masa de oxígeno y la masa de la botella. Para el 11,76\% la masa se encontrará entre 500 y $520 \mathrm{~g}$, atribuyendo la pérdida de masa a que el papel se quema. El 5,88 \% de los estudiantes piensan que la masa aumentará más de $520 \mathrm{~g}$, porque la botella se llena de aire (haciendo relación al humo) y esto hace que pese más. En el postest el 17,64\% de los estudiantes escogió respuestas incorrectas con explicaciones como que quemarse el papel la masa disminuye, porque se quema el papel o que el humo hará que el sistema pesa más.

En este sentido, teniendo en cuenta a Oñorbe y Sánchez ${ }^{52}$, se puede plantear que el aprendizaje de algunos conceptos básicos para el estudio de la química, como en este caso, los cambios químicos presentados en una reacción de combustión y la ley de conservación de la masa, no ha superado completamente el nivel memorístico o no alcanzó un nivel significativo, porque luego no es aplicable para resolver preguntas sencillas sobre estos temas.

\section{Pregunta 2. Reacción de precipitación de sulfato de bario.}

Tabla 8. Pregunta y respuestas reacción de precipitación de sulfato de bario

\begin{tabular}{|c|c|c|c|c|}
\hline \multirow{2}{*}{ ENUNCIADO } & \multirow{2}{*}{ PREGUNTA } & \multirow{2}{*}{$\begin{array}{l}\text { OPCIÓN DE } \\
\text { RESPUESTA }\end{array}$} & PRETEST & POSTEST \\
\hline & & & $\%$ & $\%$ \\
\hline \multirow{3}{*}{$\begin{array}{l}\text { Soluciones acuosas de dos sales, sul- } \\
\text { fato de sodio, } \mathrm{Na}_{2} \mathrm{SO}_{4}(\mathrm{ac}) \text {, y cloruro de } \\
\text { bario, } \mathrm{BaCl}_{2}(\mathrm{ac}) \text {, se colocan en probe- } \\
\text { tas separadas en una balanza de pla- } \\
\text { tillo superior. La masa total se registra } \\
\text { como } 140 \mathrm{~g} \text {. La solución de sulfato de } \\
\text { sodio se vierte en la solución de clo- } \\
\text { ruro de bario. Ambas probetas per- } \\
\text { manecen en una balanza. Se presenta } \\
\text { una reacción de precipitación (Özmen } \\
\& \text { Ayas, 2003). }\end{array}$} & \multirow{3}{*}{$\begin{array}{c}\text { Se presenta } \\
\text { una reacción } \\
\text { de precipi- } \\
\text { tación. ¿Cuál } \\
\text { será la lectura } \\
\text { de la masa } \\
\text { después de la } \\
\text { reacción? }\end{array}$} & Más de $140 \mathrm{~g}$ & 70,59 & 17,65 \\
\hline & & $140 \mathrm{~g}$ & 17,65 & 82,35 \\
\hline & & Menos de $140 \mathrm{~g}$ & 11,76 & 5,88 \\
\hline
\end{tabular}

Fuente: Esta investigación

52. Oñorbe y Sánchez, “La masa no se crea ni se destruye. ¿Estáis seguros?”, 1. 
El porcentaje de estudiantes que escogió la respuesta correcta en el pretest fue del $17,65 \%$ y aumento en el postest a $82,35 \%$, para estos estudiantes la masa del sistema se tiene que conservar antes y después de la reacción de precipitación. La explicación dada en el pretest para escoger esta respuesta es que las sustancias se mezclan y la masa queda igual, que la reacción no afecta el peso total. En el postest las explicaciones que se encuentran son las siguientes: la masa de reactivos es igual a la de los productos; la masa de las sustancias separadas o juntas es igual; no se aumenta ni se disminuye la cantidad de sustancia; la materia se conserva porque si se vuelven a pesar van a pesar lo mismo; solo se presenta un cambio, lo más denso se va al fondo; después de la reacción la masa es igual, solo se mezclan las dos soluciones.

Para las respuestas incorrectas en el pretest el 70,59\% de estudiantes consideró que en la reacción de precipitación aumenta la masa, justificando su respuestas de la siguiente forma: al juntar las dos soluciones pesaran más; el sólido que se forma se va al fondo y tiene más masa; lo que precipita aumenta la masa; al juntarse las masas se suman y dan más valor; y que la masa del precipitado aumenta con la masa de la solución restante. Para el $11,76 \%$ de estudiantes en la reacción de precipitación la masa se reduce, porque queda menos cantidad de solución y que al descontar la masa de las probetas la mezcla pesara menos. Por otro lado, en el postest el 17,65\% de los estudiantes considero que el sólido que se forma pesa más y que al estar todo el contenido en una probeta aumenta la masa; solo el $5,88 \%$ de estudiantes argumento que el sistema pesara menos.

\section{Pregunta 3. Reacción de formación de gases en un sistema cerrado}

Tabla 9. Preguntas y respuestas reacción de formación de gases en un sistema cerrado.

\begin{tabular}{|c|c|c|c|c|}
\hline \multirow{2}{*}{ ENUNCIADO } & \multirow{2}{*}{ PREGUNTAS } & \multirow{2}{*}{$\begin{array}{l}\text { OPCIÓN DE } \\
\text { RESPUESTA }\end{array}$} & PRETEST & POSTEST \\
\hline & & & $\%$ & $\%$ \\
\hline \multirow{6}{*}{$\begin{array}{l}\text { Carlos coloca un poco de } \\
\text { bicarbonato de sodio y un } \\
\text { vaso de jugo de naranja } \\
\text { en una bolsa de plástico } \\
\text { sellada. Nada puede entrar } \\
\text { y salir de la bolsa ni siquiera } \\
\text { los átomos. Carlos vierte el } \\
\text { jugo de naranja en la bolsa, } \\
\text { el cual al entrar en contacto } \\
\text { con el bicarbonato de sodio } \\
\text { produce burbujas y la bolsa } \\
\text { se llena de gas (Pyke \& } \\
\text { Ochsendorf, 2004). }\end{array}$} & \multirow{3}{*}{$\begin{array}{l}\text { A. ¿El número de átomos } \\
\text { en la bolsa, después de } \\
\text { que entran en contacto el } \\
\text { bicarbonato de sodio y el } \\
\text { jugo de naranja es el mismo, } \\
\text { disminuyó o aumentó? }\end{array}$} & Disminuyo & 0,00 & 0,00 \\
\hline & & Igual & 11,76 & 47,06 \\
\hline & & Aumento & 88,24 & 52,94 \\
\hline & \multirow{3}{*}{$\begin{array}{l}\text { B. ¿Después de que el } \\
\text { bicarbonato de sodio } \\
\text { y el jugo de naranja se } \\
\text { mezclan la masa de la bolsa } \\
\text { es la misma, aumentó o } \\
\text { disminuyó, comparada con } \\
\text { la condición inicial? }\end{array}$} & Disminuyo & 0,00 & 11,76 \\
\hline & & Igual & 5,88 & 41,18 \\
\hline & & Aumento & 94,12 & 47,06 \\
\hline
\end{tabular}

Fuente: Esta investigación 
En la pregunta $\mathrm{A}$, sobre el número de átomos después de la reacción entre el bicarbonato de sodio y el juego de naranja, en el pretest solo el 11,76\% de los estudiantes escogió la respuesta correcta, porcentaje que aumento a 47,06\% en el postest. Para estos estudiantes la cantidad de átomos antes y después de la reacción se mantiene igual, dando como explicación en el pretest que las sustancias tienen átomos, que solo se producen burbujas de aire que inflan la bolsa. Mientras que el postest para escoger la respuesta se consideró que se conserva la materia; se forma gas pero se conserva la masa; la bolsa está cerrada y no puede entrar ni salir átomos; al reaccionar la sustancia la masa no cambia y que la cantidad de átomos no disminuye ni aumenta.

En el pretest eligió la respuesta incorrecta el 88,24\% de los estudiantes, para quienes el número de átomos aumentó, justificando la respuesta con afirmaciones como las siguientes: se infla la bolsa al aumentar la cantidad de átomos; se formó un gas; la reacción química produjo otro compuesto; se forman burbujas al juntarse los átomos; aumenta la cantidad de aire; las sustancias se mezclan y la bolsa se llena de gas y al juntarse las sustancias aumenta la masa. Un comportamiento similar se presentó en el postest, donde el 52,94\% de estudiantes respondió que la cantidad de átomos aumenta, argumentando que se forma gas en la bolsa; se infla la bolsa porque aumenta el número de átomos; en la reacción se liberan átomos; al reaccionar las sustancias se libera gas; se forman nuevas sustancias que tienen más átomos; las sustancias se mezclan y los átomos no pueden salir y que la bolsa se llena de un producto gaseoso.

Al preguntar a los estudiantes, si la masa del sistema en el cual se realizó la prueba cambia luego de la reacción (Pregunta B), siendo la respuesta correcta que la masa se mantiene igual, en el pretest solo el 5,88\% eligió esta respuesta, en el postest este porcentaje aumento al 41,18\%. Para el 5,88\% que escogió la respuesta correcta en el pretest, la masa se mantiene igual porque solo se juntan las dos sustancias. Las explicaciones dadas en el postest para esta pregunta fueron: solo se forman nuevos productos, sigue siendo la misma masa; en la reacción las sustancias se transforman; la masa de reactivos es igual a la masa de productos y en la reacción no cambia la masa.

En el pretest un 94,12\% de estudiantes respondió equivocadamente que la masa del sistema en el que se realizó la reacción aumento, utilizando explicaciones como las siguientes: la bolsa se infló; se produce gas en la reacción; aumenta el peso del gas; se llena de oxígeno más la masa de las sustancias iniciales; se juntan las dos sustancias y la bolsa se infla; se mezclan las sustancias y se genera gas; al mezclarse las sustancias aumenta la masa; al inicio no hay reacción; y que las burbujas contienen líquido que aumenta la masa. Al igual que en la pregunta anterior, en el postest un alto porcentaje de estudiantes escogió las dos opciones de respuesta incorrecta. Así, para el 47,06\% la masa 
de la bolsa aumenta, argumentando que al aumentar los átomos aumenta la masa; al juntarse las sustancias se producen más átomos; la bolsa se llena de gas y se infla; se mezclan las dos sustancias; se forma un gas; se infla y aumenta la masa. Y para el 11,76\% el sistema de reacción pesara menos porque al llenarse de gas pesa menos y la masa no es la misma.

De los resultados del pretest y postest para conocer las ideas previas que tienen los estudiantes sobre la conservación de la masa en reacciones químicas se puede concluir lo siguiente:

A pesar de incluirse actividades de aprendizaje en el programa guía relacionadas con algunas de las pregunta formulada en el test para conocer las ideas previas, la explicación dada en el postest para la respuesta seleccionada está muy influenciada por las ideas previas; solo se incluyen algunos de los aspectos químicos estudiados en las clases; y como concluyen Landau y Lastres ${ }^{53}$ en su investigación, el cambio químico no es claramente interpretado y las concepciones alternativas son resistentes a la enseñanza.

\subsection{Test para evaluar conocimientos sobre la ley de conservación de la masa}

Con el propósito de presentar una síntesis del test de conocimientos y sus resultados, a continuación se presenta en la tabla 10 un resumen del tema evaluado en cada una de las preguntas de la prueba y en la gráfica 2 se relaciona el porcentaje de estudiantes que escogió la respuesta correcta en el pretest $y$ postest respectivamente.

Tabla 10. Resumen test evaluación de conocimiento

\begin{tabular}{|c|l|}
\hline PREGUNTA & \multicolumn{1}{c|}{ TEMA DE LA PREGUNTA } \\
\hline $1,2,6$ & Aplicación ley de conservación de la masa: balanceo de ecuaciones químicas \\
\hline $3,4,5,7,8,10,11$ & Conservación de la masa en una reacción química. \\
\hline 9,12 y 13 & Cálculos estequiométricos: interpretación de una ecuación química balanceada \\
\hline
\end{tabular}

Fuente: Esta investigación

Luego de realizar el análisis de resultados para cada una de las preguntas del test de evaluación de conocimientos sobre la ley de conservación de la masa se pueden generalizar los siguientes resultados. En cada una de las 13 preguntas del postest aumento el porcentaje de estudiantes que escogió la respuesta correcta. En el pretest un promedio del 32\% de los estudiantes seleccionó la respuesta correcta, mientras que en el postest este promedio aumentó

53. Landau y Lastres, “Cambios Químicos y Conservación de la Masa... ¿Esta todo claro?”, 1. 
al 72,85\%. Para el postest se tiene que en las preguntas 1, 2, 5 y 7 el porcentaje de acierto fue superior al $80 \%$; el porcentaje de acierto para la preguntas 3,6 , 8,10 y 11 se encuentra en el $70 \%$ y $80 \%$; la pregunta 6 es la única con porcentaje de respuesta correcta superior a 60\%, mientras que las preguntas 9,12 y 13 tienen porcentajes de acierto entre el 50\% y 60\%. El menor porcentaje de respuestas correctas para las preguntas 9,12 y 13 se puede atribuir a que en ellas se evaluaba la aplicación de cálculos estequiométricos tema de estudio que posiblemente no fue totalmente comprendido por los estudiantes.

Figura 2. Porcentaje de respuestas correctas test de conocimientos

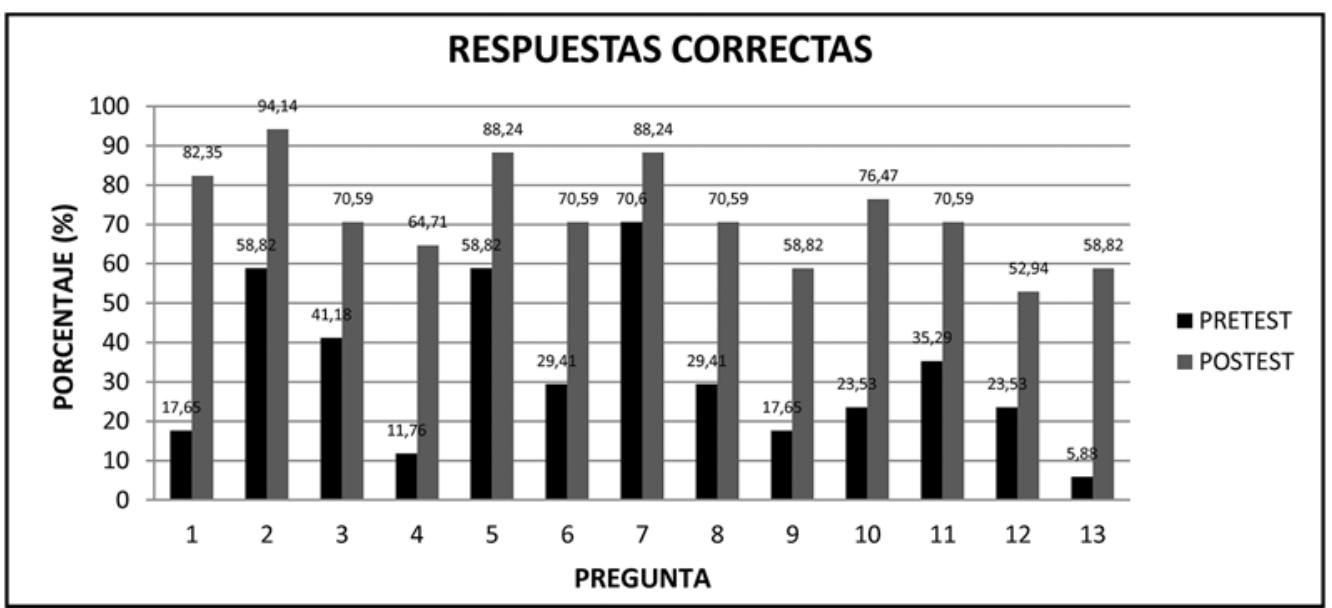

Fuente: Esta investigación

En general se puede concluir que al comparar los resultados del pretest y postest para evaluar conocimientos sobre la ley de conservación de masa, se mejoraron en el postest todos los porcentajes de selección de respuesta correcta, gracias a la realización del programa guía de actividades que desde una visión constructivista, incluía actividades de iniciación, desarrollo y finalización que integraban una secuencia de enseñanza y aprendizaje, incluyendo además la utilización pedagógica de OVAs y la realización de un laboratorio virtual de química. Con el programa guía de actividades se buscó que el estudiante asuma un papel activo y docente sea un orientador en el proceso de aprendizaje, que si bien mejoró los porcentajes de respuesta en el test de conocimientos, no permitió modificar completamente las ideas previas que tiene los estudiantes sobre el tema, como se demostró anteriormente en el análisis de resultados del test sobre conocimientos previos de la ley de conservación de la masa. 


\section{CONCLUSIONES}

El uso pedagógico de las TIC y del laboratorio virtual VLabQ mejoró el interés y la motivación de los estudiantes por las clases de química. Al integrar el LVQ a un enfoque constructivista de enseñanza de las ciencias se acercó a los estudiantes a la experimentación en química y al desarrollo de competencias en ciencias. El LVQ debe considerarse una herramienta complementaria para la enseñanza de la química, porque no proporciona una experiencia real del trabajo en un laboratorio.El test de Likert muestra que se lograron cambios de actitud entre modernamente positivas y muy positivas para los ítems analizados. El pretest para conocer las ideas previas de los estudiantes indica que los estudiantes escogen una respuesta a la pregunta formulada, basándose en estado inicial y final del sistema y en sus ideas previas; mientras que en el postest, se selecciona la respuesta utilizando estos mismos criterios complementados con algunas ideas de lo aprendido. Aumento el porcentaje de respuestas correctas del postest de conocimientos sobre la ley de conservación de la masa, indicando cierto nivel de aprendizaje de los temas estudiados. Se enriqueció la práctica docente al profundizar en el estudio del constructivismo y se adquirieron competencias para el uso pedagógico de las TIC. 


\section{REFERENCIAS BIBLIOGRÁFICAS}

Alonso, Carlos y Rodríguez, Geovany. "El laboratorio en la enseñanza de las ciencias naturales: $\iota$ plato fuerte o plato de segunda mesa?" En Educación y Ciencia, Vol. 1 No. 1 (1990), http://educacionyciencia.org/index. php/educacionyciencia/article/view/8/pdf

Astudillo, Gustavo. "Análisis del estado del arte de los objetos de aprendizaje. Revisión de su definición y sus posibilidades". Trabajo final especialización Universidad de la Plata (Buenos Aires, Argentina, 2011), http://sedici.unlp.edu.ar/bitstream/handle/10915/4212/all-0001.pdf?sequence=1

Astudillo, Marta y Chévez, Florlenis. "Valoración de las actitudes los estudiantes frente al uso de TIC". Revista Digital de la Universidad Autónoma de Chiapas. (s. f.), http://espacioimasd.unach.mx/articulos/num2/pdf/ valoracion_de_las\%20actitudes_de_estudiantes_universitarios_frente_al_uso_de_las_TIC.pdf

Baroli, Elisabeth, Laburú, Carlos y Guridi, Veronica. "Laboratorio didáctico de ciencias: caminos de investigación". Revista electrónica de enseñanza de las ciencias, Vol. 9. No 1, (2010), http://reec.uvigo.es/volumenes/ volumen9/ART6_VOL9_N1.pdf

Brown, Theodore, Lemay, Eugene, y Bursten, Bruce. Química la ciencia central, 7a edición. México: Prentice Hall Hispanoamericana S.A., 1998.

Caamaño, Aureli. "Los trabajos prácticos en ciencias". Enseñar Ciencias. Barcelona: Editorial Graó, 2003, http://www.cneq.unam.mx/programas/actuales/cursos_diplo/cursos/cursos_SEP/00/secundaria/ mat_particip_secun/02_fisica/arch_partic_fisica/S1P1.pdf

Caamaño, Aureli y Oñorbe, Ana. "La enseñanza de la química: conceptos y teorías, dificultades de aprendizaje y replanteamientos curriculares". Alambique: didáctica de las ciencias experimentales, Vol. 41 (2004), http:// biblioteca.universia.net/html_bura/ficha/params/title/ense\%C3\%B1anza-quimica-conceptos-teoriasdificultades-aprendizaje-replanteamientos-curriculares/id/ 38129531.html

Cabero, Julio. “Las TICs en la enseñanza de la química: aportaciones desde la Tecnología Educativa”. Química: vida y progreso (2007), http://tecnologiaedu.us.es/cuestionario/bibliovir/jca16.pdf

Campanario, Juan y Moya, Aida. “Cómo enseñar ciencias? Principales tendencias y propuestas”. Enseñanza de las ciencias, Vol. 17 No. 2 (1999), http://www2.uah.es/jmc/an11.pdf

Cataldi, Zulma, Chiarenza, Diego, Dominighini, Claudio y Large, Fernando. "Clasificación de laboratorios virtuales de química y propuesta de evaluación heurística. Tecnología informática aplicada a la educación." Conferencia, XIII Workshop de Investigadores en Ciencias de la Computación, Universidad Nacional del Rosario, Argentina (2011), http://hdl.handle.net/10915/19937

Chiarenza, Diego. "Las TICs en la enseñanza de la Química: Laboratorios Virtuales". Tesina en tecnología educativa. Universidad Tecnológica Nacional, Buenos Aires, Argentina (2011), http://issuu.com/diegochiarenza/docs/tesina_laboratorios_virtuales_de_qu_mica

Flores, Julia, Caballero, María y Moreira, Marco. "El laboratorio en la enseñanza de las ciencias: una visión integral en este complejo ambiente de aprendizaje”. Revista de Investigación, Vol. 33. No. 68, 2009, http:// www2.scielo.org.ve/pdf/ri/v33n68/art05.pdf

Gil Pérez, Daniel. "Contribución de la historia y de la filosofía de las ciencias al desarrollo de un modelo de enseñanza/aprendizaje como investigación”. Enseñanza de las Ciencias, Vol. 11. No. 2 (1993), http://ddd. uab.cat/pub/edlc/02124521v11n2p197.pdf

Gil Pérez, Daniel y Martínez, Joaquín. "Los programas-guía de actividades: una concreción del modelo constructivista de aprendizaje de las ciencias". Investigación en la escuela, Vol. 3 (1987), http://www. investigacionenlaescuela.es/articulos/3/R3_1.pdf

Gómez, Miguel. "Ideas y dificultades en el aprendizaje de la química". Alambique: didáctica de las ciencias experimentales, No. 7 (1996), http://alambique.grao.com/revistas/alambique/007-las-ideas-del-alumnadoen-ciencias/ideas-y-dificultades-en-el-aprendizaje-de-la-quimica 
Hernández, Carlos. "Aproximación a un estado del arte de la enseñanza de las ciencias en Colombia”. En Estados del Arte de la Investigación en Educación y Pedagogía en Colombia. Bogotá: ICFES, Colciencias, Sicolpe (2001), http://www.socolpe.org/data/public/libros/InvestigacionPedagogia/2-1Ciencias-Estado del Arte.pdf

Hierrezuelo, José, Molina, Eduardo y Yus, Rafael. "Una nueva generación de materiales curriculares para la enseñanza de la ciencia: los programas-guía de actividades”. Revista de Educación, No. 295 (1991), http://www. mecd.gob.es/dctm/revista-de-educacion/articulosre295/re29514.pdf?documentId=0901e72b813578ea

Hodson, D. "Hacia un enfoque más crítico del trabajo de laboratorio”. Enseñanza de las Ciencias, Vol. 12. No. 3 (1994), http://www.cneq.unam.mx/programas/actuales/cursos_diplo/cursos/cursos_SEP/00/primaria/ mat_particip_prim/arch_part_prim/S1P1.pdf

Jurado, Gloria, Rosero, María y Velasco Betty. “Objetos virtuales de aprendizaje (OVA) como mediadores en el proceso de aprendizaje de funciones trigonométricas”. Trabajo de grado maestría Universidad de Nariño, Pasto, Colombia, 2010.

Landau, L. y Lastres, L. “Cambios Químicos y Conservación de la Masa... ¿Esta todo claro? Enseñanza de las Ciencias. Vol.14. No. 2 (1996), http://www.raco.cat/index.php/ensenanza/article/viewFile/21446/93409

Martínez, Leonardo, Villamil, Yenny y Peña, Diana. “Actitudes favorables hacia la química a partir del enfoque Ciencia, Tecnología, Sociedad y Ambiente (CTSA)”. I Congreso Iberoamericano de Ciencia, Tecnología e Innovación CTS+I. (Junio, 2006), http://www.oei.es/memoriasctsi/mesa4/m04p53b.pdf

Martínez, Pedro. “Química de Bachillerato y laboratorios virtuales”. II jornadas sobre la enseñanza de las ciencias e ingenierías, (s.f.) http://www.murciencia.com/upload/comunicaciones/37_quimica_laboratorios_virtuales.pdf

Molina, Manuel, Carriazo, Jose y Farías, Diana. "Actitudes hacia la química de estudiantes de diferentes carreras universitarias en Colombia". Quim. Nova, Vol. 34. No. 9 (2011), http://www.scielo.br/pdf/qn/ v34n9/v34n9a32.pdf

Montoya, Diana. “Diseño e implementación de guías para el aprendizaje de la materia y sus propiedades apoyadas en herramientas virtuales". Trabajo de grado maestría Universidad Nacional de Colombia (2012), http://www.bdigital.unal.edu.co/9100/1/8410503.2012.pdf

Objetos virtuales de aprendizaje e informativos (s.f.), http://www.colombiaaprende.edu.co/ $\mathrm{html} /$ directivos/1598/article-172369.html

Oñorbe, A.y Sánchez J. “La masa no se crea ni se destruye. ¿Estáis seguros?.” Enseñanza de las Ciencias, Vol. 10. No. 2 (1992), http://www.raco.cat/index.php/Ensenanza/article/download/39817/93184

Ozmen, Haluk y Ayas, Alipasa. "Students' difficulties in understanding of the conservation of matter in open and closed-system chemical reactions". Chemistry Education:Research And Practice. Vol. 4. No. 3 (2003), http://www.uoi.gr/cerp/2003_October/pdf/040zmen.pdf

Pozo, Juan y Gómez, Mario. Aprender y enseñar ciencias, 5ta edición. Madrid: Ediciones Morata, S.L., 2006.

Pyke, Curtis y Ochsendorf, Rob. Conservation of Matter Assessment Manual. Scale-uP Research Staff (2004), http://www2.gwu.edu/ scale-up/documents/COMAManual.pdf

Ruiz, Francisco. "Modelos didácticos para la enseñanza de las ciencias naturales". Revista latinoamericana de estudios educativos, Vol. 3. No. 2 (2007), http://www.redalyc.org/articulo.oa?id=134112600004

Vidal, Gonzalo y Gonzáles, Hilda. "Evaluación pedagógica del simulador del laboratorio químico Model Chemlab”. Revista Pedagogía Universitaria, Vol. 7. No. 4 (2002), http://cvi.mes.edu.cu/peduniv/base-1/2002vol.-vii-no.-5/evaluacion-pedagogica-del-simulador-del-laboratorio-quimico-model-chemlab 